$45 \mid 2010$

Langue française, identité(s) et école(s) : le cas de la minorité catholique au Levant (milieu XIX ${ }^{\mathrm{e}}-\mathrm{XX}^{\mathrm{e}}$

siècles). Représentations identitaires et apprentissage des langues : Europe, bassin méditerranéen ( $\left.\mathrm{XV}\right|^{\mathrm{e}}-\mathrm{XX}{ }^{\mathrm{e}}$ siècles)

\title{
Les catholiques palestiniens et la langue française (1870-1950).
}

Une langue des minorités devenue minoritaire?

Karène Sanchez-Summerer

\section{OpenEdition}

Édition électronique

URL : https://journals.openedition.org/dhfles/2403

DOI : $10.4000 /$ dhfles.2403

ISSN : 2221-4038

Éditeur

Société Internationale pour l'Histoire du Français Langue Étrangère ou Seconde

Édition imprimée

Date de publication : 1 juin 2010

Pagination : $17-42$

ISSN : 0992-7654

Référence électronique

Karène Sanchez-Summerer, «Les catholiques palestiniens et la langue française (1870-1950). »,

Documents pour l'histoire du français langue étrangère ou seconde [En ligne], 45 | 2010, mis en ligne le 13 février 2014, consulté le 27 mai 2021. URL : http://journals.openedition.org/dhfles/2403 ; DOl : https:// doi.org/10.4000/dhfles.2403

Ce document a été généré automatiquement le 27 mai 2021.

(c) SIHFLES 


\title{
Les catholiques palestiniens et la langue française (1870-1950).
}

Une langue des minorités devenue minoritaire?

\author{
Karène Sanchez-Summerer
}

1 Au début du mandat britannique, le patriarche de la communauté catholique latine palestinienne, Mgr L. Barlassina, déclare en 1920 aux directeurs des écoles catholiques, à propos de l'expansion de l'anglais, qu'il leur faut «combattre la langue du protestantisme». Il pose ainsi en termes linguistiques la position de la minorité catholique. De 1870 à la fin des années 1940, l'uniforme scolaire et les bandeaux des élèves d'un des plus grands établissements pour filles, celui des sœurs de Sion, situé dans le couvent de l'Ecce Homo, sont aux couleurs de la France et du catholicisme : bleu (couleur de la Vierge), blanc (sainteté), rouge (Sacré-Cœur). Ils sont emblématiques de la relation privilégiée entre la France et le français, et les catholiques palestiniens.

2 La minorité catholique en Palestine est composée essentiellement de latins ${ }^{1}$, de melkites (grecs catholiques) et d'un très faible pourcentage de maronites, syriaques et arméniens catholiques. Selon les époques considérées ${ }^{2}$, les latins représentent entre 15 et $5 \%$ de la population palestinienne. Le terme "palestinien" indique, pour les époques ottomane et mandataire, la population musulmane et chrétienne (nous n'incluons pas la population juive non immigrée qui est palestinienne), alors que pour la période jordanienne et israélienne, nous précisons la différence entre Palestiniens (vivant en Cisjordanie et à Gaza sous régime jordanien) et Palestiniens ayant acquis la nationalité israélienne et résidant sur le sol israélien. À l'époque ottomane, la communauté catholique ne constitue pas un millet (communauté religieuse légalement protégée), contrairement aux orthodoxes et aux arméniens.

Durant la période ottomane et mandataire, les grands consulats généraux européens se servent de la langue comme d'une "arme", avec chacun leurs objectifs culturels et linguistiques. L'usage du français par rapport aux autres langues pose la question du rapport entre la langue et l'identité (Frijhoff 2006), individuelle et collective. Le français apparait comme une langue refuge, un instrument identitaire pour une minorité catholique non ethnique ${ }^{3}$, et le véhicule de pratiques linguistiques et 
culturelles spécifiques. Il sert à affirmer le rôle des catholiques au sein de l'élite et à protéger leur statut de minorité. Mais ces liens particuliers des catholiques palestiniens avec le français les place face à une critique de la part du reste de la population, qui met en doute leur enracinement dans la société. Pour les nationalistes palestiniens, le français entretient en effet une ambiguïté : il atteste une influence extérieure sur la communauté catholique face aux orthodoxes arabes palestiniens qui ont mis à l'honneur leur lutte pour le nationalisme naissant, et l'arabisation qui en a résulté, contre le poids de l'extérieur. Pourtant, en 1894, le pape Léon XIII reconnaît la valeur des rites orientaux et interdit d'inciter les fidèles de passer au rite latin (encyclique Orientalum dignitas) ; cela a des implications sur la place de l'arabe, qui devient la langue des catholiques palestiniens et supplante ainsi progressivement le français. Cependant, le remplacement du français par l'arabe s'effectue selon un processus lent, de la fin du $\mathrm{XIX}^{\mathrm{e}}$ aux années 1940 ; il est ponctué de batailles linguistiques entre les missions, qui désirent conserver le français (soutenues par les représentants de la France) et les autorités religieuses locales et romaines, qui poussent les religieux à enseigner en arabe (le catéchisme mais aussi les matières " profanes »).

4 La communauté catholique française est très attachée à la Palestine et à sa communauté catholique. Tandis que s'est jouée la perte du protectorat catholique français en Terre sainte face aux Britanniques au début des années 1920, la Palestine demeure «la plus française des terres d'Orient $»^{4}$. La communauté catholique palestinienne reste attachée au français car la France incarne le protectorat catholique qui se traduit sur le terrain par de nombreuses œuvres d'enseignement et d'assistance. Ce protectorat se développe rapidement grâce aux réformes mises en place par la Sublime Porte dès 1869, les Tanzimat. La présence continue de la France en Palestine entraîne certes une concurrence avec l'Italie. Cependant, cette concurrence est de faible importance en termes linguistiques, même si les patriarches sont italiens jusqu'aux années $1970^{5}$. Le début des années 1920, même s'il correspond à la fin officielle du protectorat français, est marqué par des symboles du lien entre pouvoir et religion catholique, en France (canonisation de Jeanne d'Arc le 16 mai 1920, achèvement des travaux et consécration de la basilique du Sacré-Cœur), mais aussi en Orient, avec la visite du cardinal Dubois, perçue comme une appropriation française du Levant.

5 Les rapports entre la langue française et la communauté catholique sont particulièrement visibles dans les écoles catholiques françaises, car l'école est appréhendée par les différents acteurs comme un lieu primordial pour ce qui concerne les liens entre langue et religion. La communauté catholique est celle qui scolarise le moins ses enfants dans les établissements impériaux puis gouvernementaux. La grande majorité d'entre eux reste éduquée dans les écoles catholiques françaises de 1860 à 1950, de 4 à 18 ans, dans les sections gratuites et payantes (ces dernières étant également ouvertes aux élèves juifs et musulmans). Les liens entre langue et religion sont souvent problématiques dans les institutions éducatives de cette zone où se concentrent de nombreux pouvoirs européens et leurs écoles, véritables fers de lance de leur langue et de leur culture nationales auprès de la population locale. Les écoles missionnaires françaises s'implantent rapidement dans les principales villes palestiniennes et cette implantation est ressentie par la population locale et présentée par les différents consuls comme un reflet de la France ${ }^{6}$. Durant la période ottomane, la qualité du réseau éducatif catholique est reconnue comme supérieure sur de nombreux points aux autres établissements (le modèle éducatif français est adopté par les 
autorités ottomanes). La loi de 1905 de séparation des Églises et de l'État n'affecte pas le soutien de la France aux communautés catholiques levantines et aux écoles missionnaires. Bien que la Première Guerre mondiale ait porté un coup sévère à ces établissements (dégradation des bâtiments et fin du français comme langue officielle et langue d'examens au début des années 1920), ce réseau se renforce, grâce au soutien réitéré de la France, face à ce qui est alors perçu par les autorités catholiques locales et romaines comme une vive concurrence protestante et anglicane. Les écoles missionnaires françaises continuent à former des cadres jusqu'aux années 1940, même si l'arabe y gagne une place de plus en plus prépondérante, supplantant le français. Après 1948, sous le régime jordanien et l'État d'Israël, ces écoles augmentent les heures d'apprentissage de l'arabe et de l'hébreu, et le français est alors appréhendé avec nostalgie, comme un bien symbolique, tandis qu'il continue d'être proposé au sein des établissements comme un « bien temporel ».

6 Il est intéressant de se pencher sur la dimension confessionnelle à travers le cas des écoles, durant la période d'élaboration de ce qui est qualifié par l'historiographie contemporaine de «constitution de l'identité nationale palestinienne». La communauté catholique, minorité au sein de la minorité chrétienne palestinienne, est assez effacée du champ historiographique pour la période qui va du XIXe à la première moitié du XXe siècle (sa situation est davantage connue après 1948 ainsi que celle de sa diaspora: Sabella 1999; O'Mahony 2008; Tsimhoni 1978 et 1993). Sa position linguistique a longtemps été délaissée par les historiens et les linguistes, tant l'arabisation a occulté la place qu'occupait le français en Palestine ottomane et mandataire. L'expression "un passé à portée de mémoire ${ }^{7}$, utilisée par Nadine Picaudou à propos du champ palestinien, explique une des raisons possibles de cette absence de l'historiographie (qui a davantage porté sur la connaissance critique du passé récent du conflit israélo-palestinien). Outre un problème temporel, un autre problème se pose : celui de la patrimonialisation, de fait parallèle à un processus de déshistorisation, puisqu'il se transforme en une forme de nostalgie du passé (préservé de manière sacralisante), qui aurait été un âge d'or de la francophonie.

7 La présente étude tente de ne pas se cantonner aux enjeux internationaux liés à la langue, mais d'analyser également dans quelle mesure la communauté catholique en a fait une langue à soi. Dans l'approche poststructuraliste, la langue ne constitue pas seulement un marqueur identitaire mais aussi un « site » de résistance, de pouvoir, de solidarité, de discrimination. Le rapport entre la langue française et les catholiques palestiniens interroge deux catégories d'analyse: celle de l'identité d'un groupe religieux et celle de l'importance de la langue face à la montée des nationalismes arabes, dans un territoire sous influence britannique.

8 L'article s'intéresse aux liens entre les catholiques palestiniens et le français ${ }^{8}$, sans prétention aucune à l'exhaustivité et essentiellement à travers les établissements francophones. Dans quelle mesure le français a-t-il constitué une langue refuge mais également une langue d'intégration dans le corps national en formation? Quels ont été les bouleversements au sein de ces établissements provoqués par l'arrivée du mandat anglais, officiellement protecteur de toutes les communautés religieuses et linguistiques de Palestine et par l'arabe promu comme langue nationale? Quels ont été les enjeux liés à l'enseignement du catéchisme et la constitution d'associations parascolaires par rapport à la diffusion de la langue et de la religion? 


\section{1. «Français et catholique » en Palestine, des synonymes}

\subsection{L'âge d'or de la francité}

Le français est un élément inséparable de la modernité à l'époque ottomane et encore à l'époque mandataire 9 . La communauté catholique est celle qui compte le plus de drogmans et qui est la plus éduquée, de manière disproportionnée par rapport à son faible nombre dans la population palestinienne. Elle est une des premières à constituer une passerelle avec les Européens. C'est également celle qui bénéficie le plus de l'enseignement des missionnaires français installés en Palestine depuis le milieu du XIXe siècle; son pourcentage au sein de l'administration, des médias et des organisations politiques est important. Plus homogène au départ (issu des différents groupes catholiques), le public des élèves de ces établissements français se diversifie en raison de leur réputation éducative et pour des raisons économiques (les élèves musulmans et juifs y sont scolarisés dans des sections payantes); le prosélytisme indirect des missionnaires ne met pas en porte à faux la communauté catholique palestinienne. Le français sert donc de «tremplin » vers ce qui est perçu par l'élite catholique palestinienne comme une modernité offerte, même si parmi les éléments de modernité, l'arabe reste la langue véhiculaire.

Le français est associé à une communauté catholique essentiellement urbaine, contrairement à la communauté orthodoxe, urbaine, rurale et arabisée plus tôt dans le système scolaire. Rome voyant jusqu'à la fin du XIX dans les rites orientaux catholiques un terrain propice à l'hérésie, le français devient également synonyme de latinisation. Les missionnaires français arrivent peu de temps après la réouverture du patriarcat latin à Jérusalem en 1847, qui soutient leur élan aux côtés de l'œEuvre des écoles d'Orient, créée en 1851. Même si la présence économique française demeure assez réduite, la langue reste synonyme de protection en vertu des accords de Mytilène (1901) et de Constantinople (1913) qui garantissent la préservation des droits des communautés catholiques au Levant. Ainsi, selon l'expression de F. Abécassis, à travers l'apprentissage du français, les écoles espèrent diffuser « du spirituel en proposant $d u$ matériel ». Toutefois, il ne s'agit pas uniquement d'un âge d'or d'une francité réservée aux élites puisqu'une grande partie de la population catholique pauvre est également éduquée en français (dans les sections gratuites de ces établissements missionnaires). Le français occupe en effet une place de choix dans une "guerre des langues » (L.-J. Calvet 1987) et dans cette "Babel linguistique sur fond d'arabe et de français dominant " (Nicault 1999: 103) qu'est Jérusalem, car elle est la langue des œuvres d'assistance les plus importantes jusqu'aux années 1930. Dans le cadre du protectorat, la communauté assiste à de nombreuses messes, cérémonies et Te Deum présidés par le consul général de France, où toute la communauté francophone est présente, même s'il n'existe pas de paroisse francophone mêlant Français et catholiques palestiniens. Les écoles missionnaires assurent une présence française très visible dans les manifestations catholiques, telle la procession des rameaux depuis le Mont des Oliviers à Jérusalem, par les bannières catholiques et françaises, les chants français, et les lieux mêmes où elles se rassemblent (départ de la procession en l'église des bénédictins français, arrivée en l'église Sainte-Anne sur la Via Dolorosa, propriété française depuis 1856). 
11 Ce réseau s'étoffe après les lois françaises sur la laïcité qui n'entament en rien la popularité de ces écoles françaises. La Palestine constitue un lieu de refuge pour les missionnaires même avant leur exil de 1905. La plupart des consuls français envoyés sur place sont d'ailleurs de fervents serviteurs de la cause catholique. En ce sens, la France en Palestine peut même apparaître comme une forme d'opposition aux lois laïques de la métropole (Bocquet 2006), d'où les passions liées aux liens entre langue et religion en Palestine pour l'opinion publique française (en Palestine, aucun établissement de la Mission laïque française n'ouvre ses portes, contrairement à son expansion ailleurs au Levant à la même époque). De grands organes de presse tels le Journal des Débats, la Revue des deux mondes et Le Temps reprennent les thèses de groupes qui soutiennent les missionnaires tels l'œEuvre de la propagation de la foi, l'œEuvre des écoles d'Orient ou le Comité des intérêts français en Orient. En 1931, les missionnaires catholiques français possèdent encore 38 écoles dans le pays et scolarisent près de $75 \%$ des élèves catholiques (plus de 5000 élèves au total), tandis que la Grande-Bretagne ne possède que 22 écoles privées, malgré le soutien du gouvernement mandataire aux écoles anglicanes.

12 Dans ce contexte, l'éducation donnée aux filles par les missionnaires françaises permet en quelque sorte de les sortir de leur « réclusion » (les sœurs françaises sont pionnières en la matière en Palestine) sans pour autant leur permettre d'accéder à des positions professionnelles importantes ${ }^{10}$. Cette éducation constitue essentiellement un atout sur le marché matrimonial, un faire-valoir. Les sœurs du Rosaire créées en 1885 (issues de l'ordre français des sœurs de Saint-Joseph) se distinguent comme le premier ordre catholique indigène, et diffèrent des autres ordres catholiques, en ce qu'elles insistent sur l'enseignement en arabe aux côtés du français et encouragent clairement leurs élèves à s'engager dans des activités médicales, sociales et d'enseignement. La femme est en effet rapidement perçue comme une "munition humaine » de l'Église face au processus de sécularisation de la société palestinienne, garante de la bonne pratique religieuse de sa famille. On note ainsi dès leur implantation une différence sensible entre les établissements pour garçons et ceux pour filles: les premiers sont plus autonomes vis-à-vis des autorités catholiques locales, tandis que les établissements de certaines sœurs, appelées par les franciscains au XIX ${ }^{\mathrm{e}}$ siècle, continuent à dépendre d'eux au niveau financier et au niveau éducatif (ils contrôlent leurs programmes). Elles proposent donc également un enseignement de l'italien.

13 Les missionnaires catholiques ne sont pas exclusivement des Français, plusieurs missionnaires catholiques allemands s'installent en Palestine dès le dernier quart du $\mathrm{XIX}^{\mathrm{e}}$ siècle. Cependant, l'influence de ces derniers est réduite (Trimbur 2007). Ils ne possèdent qu'une école pour filles, l'école Schmidt, et, en raison de leur statut de vaincues à la fin de la Première Guerre mondiale, les sœurs allemandes sont obligées d'enseigner en anglais ${ }^{11}$. L'influence allemande, plutôt associée au protestantisme, intègre le catholicisme à sa politique étrangère car il permet de contrer les ambitions françaises et confirme la Weltpolitik de Guillaume II (bien qu'émanant au départ d'une initiative privée des catholiques allemands). À la fin de la période ottomane, deux sphères d'influence s'affrontent : la francophone qui attache encore une importance au rôle de la religion comme vecteur de la langue (en effet jusqu'au début des années 1920 " catholique et français sont synonymes ${ }^{12}$ ), tandis que la nouvelle sphère anglophone propose une image détachée de cette situation traditionnelle. 


\subsection{Une langue de cœur mais pas une langue à soi} langue que par celui d'une "francisation ", cette dernière impliquant une assimilation au modèle culturel français (Laurens 2004 : 141). Le français reste la langue privilégiée jusqu'à la fin des années 1930 parmi les langues européennes parlées en Palestine par la communauté catholique, mais elle ne bénéficie pas d'une assise aussi étendue qu'au Liban et en Syrie, malgré une forte concentration d'ordres catholiques. En effet, peu d'associations littéraires et de salons sont réellement francophones. Le français constitue rarement la langue maternelle des familles des élèves, comme cela est le cas au Liban; or, la « langue de l'intime » est celle qui permet le mieux de mesurer le degré de pénétration de la langue. Par ailleurs, il y a peu de précepteurs français. Une des conséquences est donc le faible nombre d'élèves ou d'étudiants qui partent poursuivre leurs études en France ; les bourses attribuées sont minimes. Plusieurs consuls relèvent le fait que la population palestinienne est selon eux "moins éduquée » à partir des années 1930 que la population libanaise, en raison des difficultés économiques durant la période mandataire, les adolescents partant tôt pour le monde du travail. Le français reste donc avant tout un outil de reconnaissance plus qu'un outil de communication au sein de la communauté catholique, même s'il est pratiqué avec les pèlerins français, nombreux jusque dans les années 1930. Il est nécessaire de rappeler la différence entre les élites catholiques, plurilingues et francophiles, et le reste de la communauté, plus pauvre, clientèle du consulat français, bénéficiant de l'aide sanitaire et éducative des ordres catholiques, et pour qui le français est surtout synonyme d'assistance et d'une des seules possibilités de « bonne éducation ».

Un des éléments clés entre la période ottomane et le début de la période mandataire est le rôle du français dans le nationalisme naissant. L'idée promue par certains dirigeants catholiques palestiniens d'un rattachement à la Syrie (d'où l'expression de «Syrie du Sud » pour qualifier la Palestine) s'étiole après 1922, avec le début officiel du mandat britannique en Palestine. La position des catholiques est délicate, mais le militantisme national ne s'effectue pas en langue française, pour autant que l'on puisse en juger par les archives ${ }^{13}$. Les orthodoxes, qui ne bénéficient pas de structures d'éducation aussi développées que les catholiques, passent pour être davantage intégrés dans la société palestinienne, car plus proches des musulmans qui s'orientent rapidement vers le rêve d'un État panarabe, tandis que les catholiques envisagent un rattachement à une entité dont le Liban chrétien serait le centre ${ }^{14}$. On remarque cependant que les écoles catholiques ne sont jamais impliquées dans les tentatives d'émeutes contre le pouvoir britannique. La France n'est pas perçue par les catholiques palestiniens comme un contrepoids politique à la puissance mandataire. Au sein de la minorité catholique, le cas de la communauté grecque catholique (melkite) est intéressant dans la mesure où l'ensemble du clergé et des laïcs est entièrement arabe et joue un rôle moteur par rapport au nationalisme naissant (Tsimhoni 1978: 85). En ce sens, les melkites s'arrogent une grande partie de la représentativité nationale des catholiques palestiniens, sous la houlette de l'évêque Y. G. Hajjar. Ouvertement pro-français durant les premières années du mandat, ils scolarisent leurs enfants majoritairement dans les écoles missionnaires. Mais dès le milieu des années 1920, ils insistent sur le rôle de l'arabe comme langue nationale et langue de leur catholicité orientale, et certains quittent les écoles françaises.

Documents pour l'histoire du français langue étrangère ou seconde, 45 | 2010 

islamo-chrétiennes dont la première est établie à Jaffa en mars 1918, mais c'est bien l'arabe qui est la langue de ces associations. Dès le départ, ils tentent de se justifier face au nationalisme qui s'identifie de plus en plus avec l'islam car une nouvelle identité séculière ne voit pas le jour malgré les espérances d'une majorité de la population chrétienne (Tsimhoni 1978 : 77). Ils participent au mouvement en tant qu'attachés des leaders musulmans (contrairement à ces derniers, ils n'obtiennent pas de postes de direction) grâce à leur connaissance des langues étrangères, qu'ils perçoivent ouvertement comme un héritage des écoles missionnaires françaises.

L'attitude du patriarche latin de Jérusalem vis-à-vis du français révèle en partie les changements d'attitude progressifs envers la France. Tandis que durant la période ottomane il s'était montré plus favorable à l'Italie qu'à la France, cette attitude n'avait pas eu de conséquence négative sur la langue française elle-même et sur sa place au sein des écoles. Au début du mandat, il incite ses fidèles à une forme d'unité catholique voire chrétienne et se révèle plutôt pro-français face à l'attitude des Britanniques : il suggère notamment de ne pas coopérer avec leur schéma éducatif estimant qu'ils sont pro-sionistes ${ }^{15}$. Et les enfants catholiques sont en effet peu scolarisés dans les écoles gouvernementales. Le français est aussi synonyme durant cette période de langue d'affirmation internationale des catholiques palestiniens. Elle est représentée par l'Archiconfrérie du Très Saint Enfant qui réunit la plupart des écoles catholiques, créée par les frères des écoles chrétiennes en 1905, reconnue par une bulle papale en 1910, et qui a des branches dans le monde entier. Bethléem est le centre de l'association. Un journal d'abord en français puis dans d'autres langues, L'Écho de Bethléem ${ }^{16}$,se fait le porte-parole de cette catholicité palestinienne francophone et, de ce fait, a un impact non négligeable sur le réseau catholique mondial.

\section{Une langue progressivement en perte de vitesse}

\subsection{Des écoles catholiques face à de nouvelles priorités linguistiques}

18 Après les négociations de 1918, la France sort perdante en Palestine, abandonnant ses velléités d'une souveraineté territoriale. Même si le français devient peu à peu une langue minorée, la métropole entend prolonger sa présence à travers ses institutions éducatives.

En 1924, la langue française n'est plus une langue officielle aux examens ${ }^{17}$ : la priorité est clairement l'apprentissage des langues, chaque communauté étant responsable de l'enseignement des religions. Dès le milieu des années 1920, un nouveau rapport de force linguistique est en place, même si les catholiques paraissent résister plus longtemps au processus d'anglicisation qui réduit le français à la part congrue ${ }^{18}$. Le français perd ainsi progressivement de sa valeur. L'apprentissage de l'anglais, langue officielle de l'administration, de même que celui de l'arabe et de l'hébreu, garantit l'accès aux postes administratifs auxquels se destinent plusieurs élèves des établissements français catholiques, tandis que le français reste la langue de distinction des catégories sociales les plus aisées ${ }^{19}$.

Certains chefs spirituels de la communauté catholique (le patriarche latin, les vicaires patriarcaux maronite et melkite) se positionnent alors par rapport à l'utilisation de la

Documents pour l'histoire du français langue étrangère ou seconde, 45 | 2010 
langue française dans le système éducatif. Tandis qu'au début de la période mandataire, le patriarche latin L. Barlassina, élu grâce à l'influence de collèges français à Rome (Tsimhoni 1978: 85), invite les écoles catholiques à ne pas coopérer avec le schéma éducatif britannique et se montre favorable à l'influence de la France, il n'affiche plus le même soutien par la suite, sous la pression de Rome et sous le poids de l'arabe. En 1924, un évêque auxiliaire britannique (Godric Kean) est d'ailleurs mis en place au patriarcat latin. Les melkites, de tradition moins francophone, désirent un renforcement du rôle de l'arabe, parallèlement à la réitération de leur demande de la possibilité, pour leurs élèves scolarisés dans les établissements latins, d'assister aux messes melkites en arabe. Seul le patriarche maronite semble insister sur la nécessité de préserver la qualité d'un cursus francophone. Il enjoint notamment le consul français à relever le niveau du collège des frères des écoles chrétiennes de Jérusalem.

21 Face à ce nouveau rapport de force entre les langues, un certain pragmatisme est adopté dans les curricula proposés par les établissements : ces derniers augmentent les heures d'enseignement d'arabe et les diplômes professionnels sont proposés également en langue arabe, et plus seulement en français. La plupart adoptent progressivement dans les cours d'arabe une méthode de langue maternelle et non plus seulement de langue étrangère. On relève dans les rapports de ces écoles des conseils pédagogiques détaillés destinés aux enseignants d'arabe, en vue d'améliorer la qualité longtemps négligée de leur enseignement. Les méthodes utilisées reflètent la place de la langue et de la culture françaises ${ }^{20}$. Mais progressivement, derrière des images encore orientalistes d'une Palestine rurale et d'une communauté catholique palestinienne effacée, se dresse un portrait des catholiques locaux respectueux des traditions ancestrales et gardiens des lieux saints. L'indigénisation du personnel est un facteur important du renforcement de l'arabe comme langue des catholiques. Mais ceci ne s'effectue que tardivement, même si l'arabe apparaît rapidement comme la langue nationale.

Dans ce corps national en élaboration, on ne relève pas de moyens spécifiques propres aux catholiques pour préserver l'usage du français, si ce n'est un impact affectif, mais qui a des racines bien moins profondes qu'au Liban et en Syrie, et ne peut concurrencer l'effacement progressif $\mathrm{du}$ français sur la scène publique palestinienne. L'îlot linguistique francophone se réduit comme une peau de chagrin, cohabite avec l'anglais, alors langue officielle (mais pas véhiculaire) et surtout avec l'arabe (langue officielle, véhiculaire et vernaculaire dans les établissements). Les catholiques ne constituent pas du reste une communauté francophone unie. Petit à petit la langue disparaît des publicités et affiches publiques, parallèlement à l'imbrication croissante de l'arabe, de l'hébreu et de l'anglais. Même si les catholiques restent les interlocuteurs privilégiés des marchands, touristes et pèlerins français, ces derniers sont peu nombreux en Palestine. Le français est écarté progressivement des représentations officielles, et on peut évoquer à partir des années 1930 un processus de minoration linguistique. Le caractère auto-défensif de la communauté catholique n'a pas une importance majeure dans la sauvegarde de la langue française contre ce qui est alors perçu comme une menace britannique et protestante. Ainsi Jérusalem passe d'une situation de plurilinguisme à langues dominantes minoritaires (arabe et hébreu), à une situation où celles-ci acquièrent une nouvelle place dans l'espace public, parallèlement au français qui, lui, s'efface. La transmission du français, davantage assurée par un processus d'oralité (hors des écoles catholiques françaises), est peu soutenue par une diffusion du français écrit. Elle n'est pas non plus relayée par une présence plus circonscrite à un 
espace catholique (les tracts religieux, les images populaires à l'effigie de la Vierge notamment sont en arabe). La langue française est ainsi peu "performative " ${ }^{21}$, manquant totalement de relais écrits. La présence de nombreuses communautés religieuses françaises ne soutient pas réellement un écrit en français dans l'espace public, car elle est cantonnée à un cercle restreint. Les rapports sur le marché du livre en français, y compris religieux et éducatifs, confirment la perte de vitesse du français écrit (Sanchez 2009).

Les établissements catholiques français accueillent davantage d'élèves issus de communautés qui n'entretiennent pas de liens particuliers avec le français. En effet, les autres établissements missionnaires recrutent en général des élèves de leurs communautés religieuses. Mais toutes ne possédant pas d'écoles, plusieurs choisissent les établissements catholiques français. Durant cette période cruciale de la formation du Moyen-Orient moderne, au sein de ces établissements, la superposition (l'opposition voire la contradiction) des identités (Khalidi 1997) montre qu'à une communauté religieuse ne correspond pas toujours une seule langue. Pour les élèves scolarisés dans les établissements catholiques, l'arabe, le grec et l'arménien sont des langues parlées au foyer, malgré la tendance nationaliste à faire de l'arabe la seule langue. Ces différentes communautés restent attachées à leurs langues sans développer un nationalisme parallèle. Le français assure plusieurs fonctions : au niveau religieux par exemple, il n'est pas la langue de la liturgie, mais la langue des rassemblements après les célébrations, celle de la communication avec le clergé européen, des manifestations catholiques dans l'espace public et des confréries (Omoniyi 2006). Parallèlement, durant la période mandataire, certains journaux d'écoles mettent en avant une identité fondée sur la religion, la langue arabe et l'histoire locale, bien que les manuels des établissements catholiques utilisent encore en grande majorité le français. Ils montrent ainsi que les élèves sont capables "d'étudier l'Occident » (expression utilisée dans les manuels) sans perdre leur identité (c'est également ce que confirme une série d'entretiens menés auprès d'anciens élèves). Durant cette période, l'arabe devient progressivement la langue de la sécularisation face au religieux et la langue véhiculaire, tandis que la prévalence du français, langue étrangère, devient plus faible, tout comme son potentiel à relier les locuteurs entre eux ${ }^{22}$.

24 Tandis que les visites prestigieuses de catholiques français à ces établissements perdurent (celle de l'abbé Pierre en 1959 par exemple), et que la France continue à décorer les missionnaires (palmes académiques et légions d'honneur en 1955 et 1963), les diplômes sont désormais remis par le gouverneur jordanien lors de cérémonies en arabe. Il s'agit alors pour la communauté catholique de trouver une voie combinant identité catholique et identité nationale.

\subsection{Français, arabe, identité catholique palestinienne et identité nationale}

25 En dépit de cette protection française sporadique, les catholiques s'investissent davantage dans le nationalisme, tandis que parallèlement, la langue arabe vient renforcer l'orientation de l'identité palestinienne sur un socle plus musulman.

Il y a effectivement une forme de correspondance entre la notion d'identité linguistique et celle d'identité religieuse, puisque le nombre d'élèves musulmans dans les écoles catholiques reste minoritaire (il oscille entre 8 et $15 \%$ durant les périodes ottomane et 
mandataire, bien que le français ne soit pas une langue de conversion - le prosélytisme reste très marginal). L'arabe, longtemps associé au kûttab (écoles rattachées aux mosquées) et à l'enseignement du clergé local, synonyme de médiocrité, est mis à l'honneur avec la politique du pape Léon XIII qui privilégie les chrétiens orientaux (encyclique Orientalium dignitas de 1894) et impose le respect des rites orientaux. Cela implique en Palestine que la langue locale est introduite dans les établissements des missionnaires dans le cadre de l'enseignement religieux. Face au phénomène progressif de l'arabisation dans les années 1930, le patriarcat invite les établissements pour garçons à améliorer leur enseignement de la religion et à dispenser les cours de catéchisme uniquement en arabe. D'autre part, toujours en faveur de l'arabisation du clergé local, le patriarcat s'oppose à l'envoi de séminaristes de langue maternelle arabe en France pour leur formation, et vitupère contre les missionnaires français qui sont tenus pour responsables de ces départs, "au lieu de faire fructifier cet important patrimoine de la langue qui devrait plus tard leur servir à faire tant de bien à leurs compatriotes ${ }^{23}$. Les autorités religieuses palestiniennes et romaines estiment que la langue est le moteur des pratiques religieuses, et elles reprochent aux écoles catholiques françaises de faire moins de cas de l'enseignement religieux que de celui de la langue française.

Pour les missionnaires français, l'arabe liturgique est jugé trop difficile, tandis que la langue française est celle de la "réunion au catholicisme », les prêtres palestiniens sont mentionnés par les religieux français comme ne sachant pas enseigner le catéchisme. Les antagonismes persistent également entre les missionnaires français et les franciscain ${ }^{24}$. Dès la fin de la Première Guerre mondiale, les représentants de la France en Palestine doivent lutter contre l'internationalisation et la dénationalisation des communautés religieuses orchestrée par Rome (comme la Propagation de la foi qui quitte Lyon et s'installe à Rome; Trimbur 2007: 6). Pourtant, les établissements missionnaires français tiennent compte, même tardivement, de cette arabisation. Durant les années 1930, les rapports de visite de ces établissements exposent une réflexion pédagogique sur l'enseignement $d u$ catéchisme en arabe. Outre l'indigénisation du personnel enseignant, Vatican II et la tenue des messes en arabe renforcent et confirment la place de l'arabe comme langue des catholiques palestiniens.

Ces établissements désirent en effet avoir une part active dans le processus d'identité collective palestinienne. Sabella (1999: 2) évoque la part importante qu'occupe par exemple l'établissement des frères dans ce qu'il nomme «the fabric of the Palestinian Jerusalem community ", avec une éducation présentée comme une tradition familiale de père en fils, chrétienne et musulmane. Dans ce processus, l'arabe est au premier plan et à mesure que le nationalisme et l'arabisation progressent en Palestine, le français régresse. On repère dans ces établissements une tentative pour transmettre une conscience arabe à travers l'amour de la langue et de la littérature "nationales » et l'évocation d'une histoire arabe (qui n'est pas enseignée comme une histoire islamique). Les melkites jouent un rôle moteur dans ce processus d'arabisation, qu'on ne retrouve pas à un tel degré parmi les autres communautés catholiques palestiniennes. Cela entraîne une division entre les catholiques latins palestiniens et les melkites : ces derniers sont accusés par le patriarcat de céder au nationalisme arabe et au risque d'expulsion d'Orient des religieux européens. Même si les relations entre langue et religion sont perçues à travers le prisme du nationalisme par les institutions romaines - «le nationalisme régional exige actuellement la réhabilitation de la langue arabe $»^{25}$ - les établissements catholiques latins ne s'impliquent pas dans les grèves 
contre les Britanniques (1936-1939). Cependant, ils ne sont jamais pris pour cible par les manifestants ${ }^{26}$.

Durant la période mandataire, le patriarcat latin réitère ses accusations contre les œuvres postscolaires protestantes associées à l'anglais. Il se montre « très préoccupé de sauver de l'emprise protestante la jeunesse de Jérusalem», et fonde notamment l'association du Sacré-Cœur le 21 septembre 1924, dans la chapelle du collège des frères ${ }^{27}$. Les associations rendent les établissements français catholiques visibles dans les différentes manifestations religieuses et politiques, prenant ainsi une part active à l'Action catholique ${ }^{28}$. Si l'instruction religieuse en elle-même est assez faible, c'est bien la culture qui participe à la transmission d'un savoir-être chrétien et en même temps décline un savoir-être ensemble à la française et contribue à assurer une visibilité de la communauté catholique dans les villes ${ }^{29}$. Le français prend également une dimension internationale à travers la communauté catholique par le biais du journal L'Écho de Bethléem de l'Archiconfrérie du Très Saint Enfant. Celle-ci constitue en effet le centre de toutes les associations de même nom; elle regroupe en quelques années des centaines de milliers d'adhérents à travers les écoles des frères des écoles chrétiennes du monde, assure davantage de visibilité à la ville de Bethléem et promeut l'image d'une Palestine catholique spirituellement dynamique. La plupart des activités organisées se déroulent en français lorsqu'elles sont encadrées par des religieux français; il existe alors peu d'associations catholiques semblables utilisant uniquement l'arabe. Ces associations contribuent à renforcer la pratique de la langue et les activités culturelles francophones ainsi que la camaraderie, tout en étant basées sur des devoirs chrétiens. Les calendriers de ces établissements suivent une temporalité double: française et catholique. Lors de l'ouverture des cérémonies des associations, des remises de diplômes, de réceptions de personnalités diverses, on relève un certain mélange des genres : l'hymne papal est entonné ainsi que La Marseillaise, les discours sont prononcés en français, tandis que les interventions sur des enjeux plus locaux le sont en arabe. De même, dans les salles de réception et des associations de ces établissements, le drapeau du patriarcat latin trône à côté du drapeau français; autant d'indices matériels qui reflètent la dualité présente dans ces établissements. Pourtant un glissement significatif s'opère au sein des écoles catholiques durant les années 1940, les combats de 1948 et l'arrivée des réfugiés. Tout en maintenant les associations communautaires, les établissements catholiques français s'impliquent dans l'assistance à l'ensemble de la société palestinienne, perpétuant une tradition catholique établie depuis 1880 (juste après le YMCA, notamment avec l'association Saint Vincent de Paul). Ainsi la langue française est associée à des actions charitables qui ont un réel impact sur la société, notamment lors du tremblement de terre de 1927 et des combats de 1948. Le collège des frères de Caiffa devient par exemple une antenne du Comité arabe pour la distribution de vivres. Le collège de Nazareth s'adresse aussi à des laissés pour comptes, musulmans et chrétiens, avec son centre de rééducation des enfants abandonnés « ou dont le milieu est jugé mauvais par la Direction des Affaires sociales $»^{30}$.

30 Au début des années 1950, le nationalisme palestinien s'identifie de plus en plus avec l'islam tandis que la papauté met l'accent sur la transnationalité et les valeurs universelles du catholicisme. Les établissements catholiques françaises pour garçons continuent à scolariser un nombre important de musulmans (ces derniers y deviennent majoritaires par la suite), malgré les attaques de certains groupes musulmans basés en Jordanie. L'éducation dispensée par ces établissements est en effet tenue pour responsable par certains musulmans du processus d'émigration qui touche la 
communauté chrétienne (à Jérusalem, la communauté catholique est affectée plus ou moins dans les mêmes proportions que les communautés orthodoxe et musulmane). Parce que français, ces établissements sont alors présentés comme «extérieurs à la nation musulmane $\aleph^{31}$, opinion qui perdure durant la période de boycottage de la France par la Jordanie en raison de la guerre d'Algérie. Avant la loi scolaire de 1955, les discussions portent sur le vendredi proposé comme unique jour chômé à la place du dimanche. Cependant, en Palestine même, les visites officielles du Roi et de la Reine, et de certains dignitaires musulmans (tels le Sheikh Al Jabari d'Hébron et le mufti de Jérusalem, ancien élève des frères) soutiennent l'éducation de ces établissements, qu'ils estiment loyaux envers le régime.

\section{Conclusion}

31 Lors de l'émigration palestinienne de 1948, la langue française sert de passeport et de garantie éducative à la communauté catholique, mais peu de catholiques palestiniens émigrent vers la France: au rapprochement culturel ne correspond pas un rapprochement géographique. L'émi-gration se concentre plutôt vers les États-Unis (où certains membres de la communauté avaient commencé à émigrer auparavant), le Liban et la Jordanie. Ainsi, tandis qu'à Amman on prénomme « colline du bonjour » le quartier où viennent habiter de nombreux catholiques palestiniens, l'anglais devient progressivement la langue de communication avec la diaspora, et le français devient une « langue en jachère » (Laponce 2006).

32 À travers ces établissements catholiques français apparaît le changement de stratégie dans la politique linguistique et culturelle française en Palestine: une politique culturelle qui se confond par certains aspects avec la politique religieuse. Au début des années 1940, le consul de France A. Outrey continue de rejeter tout soutien de Londres aux fondations religieuses catholiques. Mais la transition que ces écoles connaissent au début des années 1950 correspond aussi à la transition de la Palestine à une plus large échelle, qui cesse d'être uniquement pour les catholiques français la «Terre sainte chrétienne », pour revêtir l'image de la " Palestine déchirée ».

Au début des années 1930, sous le consulat du vicomte d'Aumale, les écoles catholiques françaises se tournent davantage vers le public juif séfarade, généralement francophone, estimant qu'il représente une clientèle potentielle nombreuse. Après la création de l'État d'Israël, certaines de ces écoles catholiques, désormais en territoire israélien, s'adressent plus clairement encore au public juif, majoritairement séfa-rade. Le catholicisme français tente alors une réorientation, perceptible notamment au sein des établissements des pères et des sœurs de Sion (circulaire de 1951 des sœurs de Sion par exemple: "nous avons à combattre l'antisémitisme condamné par le pape [...] positivement, nous avons le devoir de nous efforcer d'intéresser les chrétiens à la cause d'Israël »). Plus réservés par rapport à la "cause d'Israël », les frères des écoles chrétiennes et les sœurs de Saint-Joseph solennisent les fêtes juives, donnent congés aux élèves tandis que, dans le nouvel État, les attaques d'associations juives et sionistes contre les écoles catholiques françaises se multiplient, au début des années 1950 et jusqu'en 1963. Elles sont appelées "écoles de la Mission" (elles apparaissent dans les argumentaires comme relevant de la même catégorie que les établissements protestants anglophones); les associations juives et sionistes leur reprochent leur 
prosélytisme religieux et incitent les familles juives à envoyer leurs enfants dans des écoles juives.

Dans les territoires contrôlés par la Jordanie, le consulat français continue à soutenir ces établissements catholiques reconnus par le régime, tandis que la crise des vocations en France, qui a débuté quelques décennies plus tôt, fait diminuer le nombre d'enseignants religieux français venus en Terre sainte. Avec les actions des associations charitables catholiques, la langue française prend une autre valeur pour les réfugiés musulmans et chrétiens. Ce rôle perdure après 1967, si bien que même si les écoles catholiques sont encore considérées par le patriarche latin en 1957, comme « une des conditions de base du catholicisme en Terre sainte", elles ne constituent pas pour autant des ghettos communautaires catholiques.

\section{BIBLIOGRAPHIE}

BARRÈS, Maurice (1923). Enquête aux pays du Levant. Paris : Plon.

BAUMGARTEN, Helga (2004). The politicization of the Muslim- Christian relations in the Palestinian National Movement, in John Bunzl (ed.), Islam, Judaism and the political role of the religions in the Middle East. Gainesville : University Press of Florida, 75-96.

BOURDIEU, Pierre (1982). Ce que parler veut dire. Paris : Fayard.

BOCQUET, Jérôme (2006). Le rôle des missions catholiques dans la fondation d'un nouveau réseau d'institutions éducatives au Moyen-Orient arabe, in Pierre-Jean Luizard (éd.), Le choc colonial et l'islam. Les politiques religieuses des puissances coloniales en terres d'islam. Paris : La Découverte, 327-342.

CALVET, Louis-Jean (1987). La guerre des langues et les politiques linguistiques. Paris : Payot.

FRIJHOFF, Willem (2006). « Langues et religions : l'enjeu identitaire », Documents pour l'histoire du français langue étrangère ou seconde, 37 : 191-197.

GELVIN, James L. (2005). The modern Middle East : a history. New York : Oxford University Press.

KHALIDI, Rashid (1997). Palestinian identity. The construction of modern national consciousness. New York : Columbia University Press.

LAPONCE, Jean A. (2006). Loi de Babel et autres régularités des rapports entre langue et politique. Montréal : Presses de l'Université Laval.

LAURENS, Henry (2004). Orientales III. Parcours et situations. Paris : CNRS Éditions.

MYHILL, John (2006). Language, religion and national identity in Europe and the Middle East. Amsterdam : John Benjamins.

NICAULT, Catherine (1999). Jérusalem 1850-1948, des Ottomans aux Anglais, entre coexistence spirituelle et déchirure politique. Paris : Éditions Autrement.

OKKENHAUG, Inger Marie (2002). The quality of heroic living, of high endeavour and adventure : Anglican mission, women and education in Palestine (1888-1948). Leiden : Brill. 
O'MAHONY, Anthony (2008). Les chrétiens palestiniens : politique, droit et société, 1917-1948, in Dominique Trimbur et Ran Aaronsohn, De Balfour à Ben Gourion, les puissances européennes et la Palestine, 1917-1948, Paris : CNRS Éditions, 357-404.

OMONIYI, Tope \& FISHMAN, Joshua A. (eds) (2006). Explorations in the sociology of language and religion. Amsterdam : John Benjamins.

PAVLENKO, Aneta \& BLACKLEDGE, Adrian (2004). Negociation of identities in multilingual contexts. Clevedon : Multilingual Matters.

RIFFIER, Jean (2000). Les œuvres françaises en Syrie (1860-1923). Paris : L'Harmattan.

SABELLA, Bernard (1999). A century apart : Palestinian Christians and their churches. From awakening to nation building, in Out of Jerusalem, Jérusalem : Afief Safieh.

SANCHEZ, Karène (2006). « Langue(s) et religion(s) en Palestine mandataire au sein d'institutions éducatives catholiques. Établissements des frères des écoles chrétiennes et sœurs de Saint-Joseph de l'Apparition ", Documents pour l'histoire du français langue étrangère ou seconde, 37 : 93-132.

- (2009). Politiques, éducation et identités linguistiques : Le collège des frères des écoles chrétiennes de Jérusalem (1922-1939). Utrecht : LOT.

TRIMBUR, Dominique (2007). « Catholiques français et allemands en Palestine, XIX ${ }^{\mathrm{e}}$-XX ${ }^{\mathrm{e}}$ siècles », Bulletin du Centre de recherche français de Jérusalem, 18 : 92-106.

TSIMHONI, Daphne (1978). The Arab christians and the Palestinian Arab National Movement during the formative stage, in Gabriel Ben-Dor (dir.), The Palestinians and the Middle East Conflict, Ramat Gan : Turtledove Publishing : 73-98.

- (1993). Christian communities in Jerusalem and the West Bank since 1948 : an historical, social and political study. Westport : Praeger.

\section{NOTES}

1. APLJ (Archives du patriarcat latin de Jérusalem) et PRO (Public Record Office), Blue Books.

2. Les chrétiens représentent $13 \%$ de la population palestinienne en $1890,10 \%$ au début du mandat, 9 \% en 1931 selon le recensement des Britanniques, 7,6 \% en 1948 (Sabella 1999).

3. Les minorités religieuses dites ethniques, telles la communauté grecque ou la communauté arménienne de Palestine, parlent la langue de leur « ethnie » (le grec et l'arménien), tandis que la communauté catholique a comme langue maternelle l'arabe.

4. Annuaire de l'Cuvre d'Orient, Bulletin de 1919.

5. La langue italienne est davantage présente dans les écoles tenues par les franciscains jusqu'au début du XXe siècle, qui scolarisent une population chrétienne plus pauvre.

6. AMAE (Archives du ministère des Affaires étrangères), Nantes, série B, Consulat de France à Jérusalem (période ottomane). En 1860, une des premières actions du curé français Bost, venu à Naplouse à la suite de la conversion de jeunes orthodoxes au catholicisme, est de mettre le pavillon de la France au-dessus du bâtiment où il s'implante, en même temps que les cloches (les premières à Naplouse). Voir J. A. Jaussen (1927). Coutumes palestiniennes, Naplouse et son district, Paris : P. Geuthner.

7. Nadine Picaudou : intervention à l'université de Birzeit, «État de la recherche sur la Palestine en France ", MAE - Fonds d'Alembert, 21 novembre 2009. Elle y évoque la "non-clôture» du passé, la difficulté de le mettre à distance pour en faire une étude plus critique. Le rôle de la diaspora et les enjeux de la France au Liban puis sa position par rapport à la création de l'État 
d'Israël peuvent être également considérés comme des " prismes déformants ». Les études sur la Palestine en France ont surtout porté sur les pratiques de l'espace et les mutations de la territorialité car elles correspondaient, au niveau épistémologique, à une volonté de sortir la Palestine de son exceptionnalité. Selon N. Picaudou, cette fascination du présent a entraîné un "déficit sur l'histoire ». Cette recherche en France s'est surtout effectuée à travers deux catégories d'analyse : le conflit et le nationalisme. Il en a résulté un manque d'intérêt pour le français et les francophones en Palestine, alors que cette étude a été amplement développée pour d'autres zones levantines, telles que l'Égypte (F. Abécassis), le Liban (C. Verdeil, E. Möller) et la Syrie (J. Bocquet).

8. Pour la question du maintien et de la diffusion de la langue française et de la religion catholique dans deux établissements catholiques (la langue française étant vecteur de la religion catholique et la religion catholique vecteur de la langue française), voir Sanchez (2006). D'aucuns pourront s'interroger sur la pertinence de limiter l'analyse à un groupe confessionnel minoritaire, plutôt qu'à celui de la classe sociale. Le critère de la classe sociale est valide dans le cas de la société palestinienne de cette époque, car élites chrétiennes et musulmanes scolarisent leurs enfants plus ou moins sur les mêmes bancs scolaires. Cependant, si l'on s'attache à l'analyse des effectifs, on relève de nettes différences parmi les élites, et la différenciation s'accentue au fil de la période puisque les catholiques restent très majoritairement élèves des écoles missionnaires françaises. Un des éléments justifiant cette démarche réside dans la longue protection que la France exerce sur cette communauté catholique palestinienne.

9. Cette notion de modernité est problématique. Voir Gelvin (2005). Michael Marten quant à lui distingue quatre types de modernité en Palestine mandataire : celle de l'Occident, celle apportée par les missionnaires, celle du sionisme et celle des élites arabes locales; voir M. Marten (université de Stirling), allocution au WOCMES III, 19-24 juillet 2010, université autonome de Barcelone, "The negociation of competing modernities in Palestinian Spaces" (sous presse).

10. Nous reviendrons dans des recherches ultérieures sur la notion de role model dans les écoles catholiques pour filles puisque la plupart des maîtresses restent des sœurs pour la période considérée, contrairement aux maîtresses laïques des écoles protestantes, femmes souvent célibataires qui atteignent une haute position dans la société et sont des précurseurs dans les domaines de la santé notamment (I. M. Okkenhaug, 2004).

11. Voir Sahar Hamouda (2010). Once upon a time in Jerusalem. Garnet Publishing : 73.

12. ACC (Archives du collège de Caiffa), frère visiteur Hugonis arrivant à Caiffa le $1^{\mathrm{er}}$ avril 1883.

13. Aucune trace de tract, de comptes rendus de réunions en français. Même si les élites libanaises favorables au rattachement de la Syrie du Sud s'expriment la plupart du temps en français, les échanges avec les leaders palestiniens s'effectuent en arabe.

14. La majorité catholique maronite libanaise a un autre rapport plus étroit avec le français, il est vrai, depuis 1860 et l'intervention de la France, mais pense son rapport à la religion différemment, en fonction de son caractère ethnique qui l'incline davantage à une forme de nationalisme territorial (Myhill 2006), ce qui ne tend pas à favoriser un rapprochement aisé avec les latins palestiniens. En termes spirituels cependant, la communauté catholique palestinienne ne se pense pas comme une périphérie de la Syrie du Sud : elle se considère comme plus proche du centre spirituel catholique, le Saint Sépulcre de Jérusalem.

15. Position remarquable dans le journal diocésain La Palestine.

16. ACB (Archives du collège de Bethléem), Archiconfrérie du Très Saint Enfant et APLJ.

17. AMAE, E 312/ 32, 26 avril 1924.

18. Les séminaires melkites de Nazareth et de Sainte-Anne à Jérusalem sont maintenus en langue française par exemple; la communauté semble moins sensible à la politique culturelle britannique que dans d'autres zones du Levant, le British Council s'installant relativement tard en Palestine. 
19. Les communautés religieuses ne sont pas les seuls instruments du Quai d'Orsay qui se sert notamment des établissements de la Mission laïque française (là où ils existent) et de ceux de l'Alliance israélite universelle.

20. Archives de la Custodie de Terre sainte et archives des établissements des frères des écoles chrétiennes et des sœurs de Sion notamment.

21. Selon Bourdieu (1982), une langue est d'autant plus «performative » [sic] que son locuteur occupe une position d'autorité.

22. Après plusieurs années de faible activité en raison de l'absence de nombreux anciens élèves depuis 1948, le nouveau Club de la Salle sera créé au collège des frères en 1965. La composition de ses membres fondateurs indique Aref el Aref (ancien maire de Jérusalem), Issa Boullata (sousdirecteur du collège St George) et d'autres grands noms, mais qui ne sont pas spécialement francophones.

23. AMG, Rapport sur le catéchisme et la formation religieuse, 1932.

24. Significativement, le premier livre pour l'enseignement du catéchisme est un catéchisme en arabe et italien imprimé dans la première imprimerie du pays, des frères franciscains, importée dès 1848.

25. AMG, 13 mai 1898, la Sainte Congrégation pour l'Église Orientale, circulaire « aux maisons généralices et à la direction des œuvres scolaires dans le Proche-Orient arabe ».

26. ACC; le 2 novembre 1929, à Caiffa, lors d'une manifestation contre la Déclaration Balfour pour en faveur d'un foyer national juif, les manifestants s'arrêtent devant le collège des frères des écoles chrétiennes, entonnent la Marseillaise en arabe et crient «Vive la France ! a avant de poursuivre leur marche.

27. Revue Jerusalem, Archives assomptionnistes, p. 281.

28. L'Action catholique en métropole a pour buts de constituer de nouveaux outils pour christianiser ou entretenir la foi et apporter la doctrine humaniste et sociale de l'Église.

29. À titre d'exemples : procession de Caiffa de 1933 pour la pluie ; réunion de la Jeunesse intellectuelle chrétienne le 12 décembre 1943.

30. ACC et ACN (Archives du collège de Nazareth).

31. AMAE, Nantes, C28, Campagne de presse contre les écoles françaises; d'autres journaux vantent leurs « efforts au service de la langue nationale »; (Sanchez 2009 : 318).

\section{RÉSUMÉS}

L'article aborde les relations entre la communauté catholique palestinienne et la langue française en Palestine ottomane et mandataire (1870-1950) à travers les écoles catholiques françaises ainsi que le processus qui aboutit à la minorisation de cette langue des minorités. Souvent accusés d'être des missionnaires français trop patriotes par les autorités religieuses, elles proposent un enseignement et une éducation chrétiens à des élèves issus de diverses communautés religieuses. Avec la fin du protectorat catholique de la France en Palestine, après la Première Guerre mondiale, la langue française reste dans une certaine mesure un vecteur de la religion catholique et la religion catholique un vecteur de la langue française au sein de ces établissements. L'arabe y devient (cependant) de plus en plus important, parallèlement à l'arabisation de la société et au nationalisme, mais elles continuent à former des cadres francophones. Après 1948, l'apprentissage du français revêt une fonction davantage symbolique et est proposé comme un 
bien temporel et non spirituel. Les actions d'assistance des écoles contribuent à diffuser une image positive de la langue.

This article approaches the relations between the Palestinian Catholic community and the French language in Ottoman and British Palestine (1870-1950) through the French Catholic schools, and the process that resulted in the minorization of this minority language. Often accused of French patriotism by the religious authorities, they propose a Christian education to pupils from various religious communities. With the end of the French Catholic Protectorate in Palestine after the First World War, French stays to a certain extent a vector of catholic religion and vice versa in these schools. Arabic becomes more and more important, but they continue to train francophone executives. After 1948, the learning of French language becomes more symbolic, proposed as a temporal good and not as a spiritual one any more. The relief actions of these schools contribute to spread a positive image of the French language.

\section{INDEX}

Mots-clés : catholiques palestiniens, établissements scolaires confessionnels, identités confessionnelles, missions catholiques françaises, Palestine mandataire britannique, politiques linguistiques et religieuses, présence française en Palestine

Keywords : British Palestine, catholic French missions, catholics in Palestine, denominational identities, denominational schools, French presence in Palestine, linguistic and religious policies

\section{AUTEUR}

\section{KARÈNE SANCHEZ-SUMMERER}

Université de Leyde, Pays-Bas

K.Sanchez@hum.leidenuniv.nl 